\title{
HHV8-Positive Multicentric Castleman Disease
}

National Cancer Institute

\section{Source}

National Cancer Institute. HHV8-Positive Multicentric Castleman Disease. NCI

Thesaurus. Code C150404.

A systemic form of angiofollicular hyperplasia associated with HHV8 infection. 